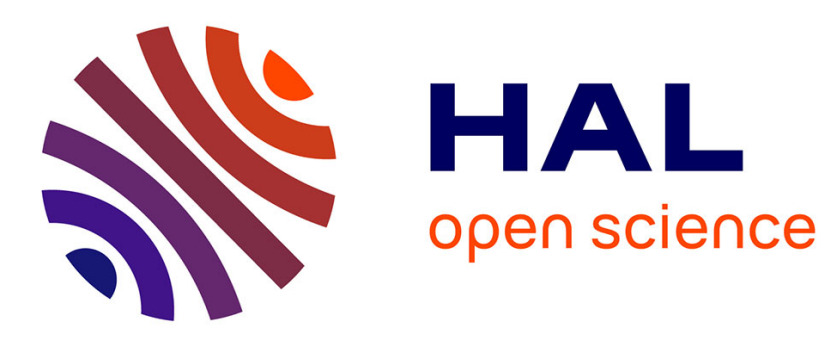

\title{
VOLTAGE CONTRAST
}

Pascal Girard

\section{To cite this version:}

Pascal Girard. VOLTAGE CONTRAST. Journal de Physique IV Proceedings, 1991, 01 (C6), pp.C6259-C6-270. 10.1051/jp4:1991640 . jpa-00250726

\section{HAL Id: jpa-00250726 https://hal.science/jpa-00250726}

Submitted on 1 Jan 1991

HAL is a multi-disciplinary open access archive for the deposit and dissemination of scientific research documents, whether they are published or not. The documents may come from teaching and research institutions in France or abroad, or from public or private research centers.
L'archive ouverte pluridisciplinaire HAL, est destinée au dépôt et à la diffusion de documents scientifiques de niveau recherche, publiés ou non, émanant des établissements d'enseignement et de recherche français ou étrangers, des laboratoires publics ou privés. 


\title{
VOLTAGE CONTRAST
}

\section{P. GIRARD}

Laboratoire d'Informatique, de Robotique et de Microélectronique de Montpellier (UA CNRS D01480), Université de Montpellier II, Sciences et Techniques du Languedoc, Place Eugène Bataillon, F-34095 Montpellier Cedex 5, France

\begin{abstract}
In recent years, a lot of research has been carried out into voltage contrast which is now widely used in both failure analysis and the debugging of innovative integrated circuits. The aim of this paper is to review past developments in this field and its present and future ability to keep pace with the progress in microelectronics.

Since secondary electrons are involved in this phenomenon, a brief summary of their properties is drawn up. The two physical effects produced, i.e. the local field effects and the secondary energy shifts, are then examined. They give rise respectively to: $i)$ the qualitative voltage contrast pictures easily seen inside any standard scanning electron microscope, and ii) the quantization of local voltages using the electron beam as an electrical probe where, however, a spectrometer is required. Then, dynamic voltage contrast is examined and the conditions for extending this method to normally working integrated circuits are considered. On the one hand, the resultant testing of conductors buried under insulator offers new and very important capacities. On the other hand, some limitations in the very high frequency domain are discussed.

Electron beam testing must not, by definition, change the working conditions of the Integrated Circuit (IC). The physical conditions of the disruption of ICs by electron beam irradiations are presented. The use of the electron beam as an active probe is then discussed.

Electron beam testing techniques and their implementation on nowadays ICs are then briefly reviewed. So, both stroboscopic observations and local voltage quantization are available today. Other probing techniques are discussed which can be reckoned on as complementary methods, especially in the case of very high speed circuits such as HEMTs.

In conclusion, voltage contrast is now of a mature age, but the extension towards future microelectronics also presupposes an extension in the domain of in situ testing methods and techniques.
\end{abstract}




\section{I) Introduction.}

Due to the extended area covered by Scanning Electron Microscopy (SEM), it is becoming a commonly used technique today. Among the different SEM contrasts, such as those related to material, topography, crystalline orientations, local voltages and magnetic domains, particular attention has been paid, in recent years, to voltage contrast. Although it was first pointed out many years ago $/ 1,2 /$, it only became the object of intensive research in the 80's, mainly in order to keep pace with developments in Very Large Scale Integration (VLSI). It can give rise to different types of information.

Firstly, voltages appearing on surface or buried lines are visualized on SEM pictures. The brilliance level is reversely proportional to the positive voltage magnitude.

Secondly, the implementation of a secondary electron spectrometer on Scanning Electron Microscopes has given access to local voltage quantization. In this case the beam does not scan the circuit anymore, but it is focussed on the area of interest.

In this paper, the main physical aspects related to voltage contrast are reviewed. After a brief introduction to secondary electron emission which generates voltage contrast, we go into voltage contrast in more detail. First of all, the origins of this contrast along with the voltage quantization are examined in the case of static voltages. Then, the methods of observation and quantization on normally working Integrated Circuits (ICs) are taken into account. Another important point, that of lines buried under insulator, is also examined.

Since, by definition, the electron beam testing of circuits must not be invasive, the possible disruptions induced by electron beam irradiations are examined. In addition, the use of the active role of the electrons in the injection of logic levels is considered.

Today, e-beam testing methods are becoming powerful, but, in the high frequency range, i.e. higher than some Gigahertz, they suffer from some intrinsic limitations. Due to the fact that electrical circuit simulations are limited to linear small signal operations, a need for quantitative observations inside $\mathrm{GHz}$ ICs is still present. Fortunately, complementary techniques are provided in the form of optical methods.

The paper is then drawn to a close with a conclusion.

\section{II) Physical phenomenon related to secondary electrons emission.}

Among the electrons re-emitted by a metal or an insulator under low primary energy irradiations (.5-10keV), the most numerous are the backscattered and secondary electrons $13,4 \%$. The backscattered re-emission coefficient $\eta$ depends mainly on the atomic number $/ 5 /$ and the angle of incidence of the primary electrons. Since their energy is not too far from the primary electron energy, they become insensitive to local voltages around the emission point. This is not true for the Secondary Electrons (SE) whose energy eW is, by definition, limited to a maximum value of $50 \mathrm{eV}$. Thus, local voltages at or around the impact point give rise to a contrast which is called the voltage contrast $/ 1 /$. In short, positive and negative surface lines appear in black and white respectively. It is important to remember that: $i)$ the distribution of the secondary electron energy $\mathrm{eW}$ presents an asymmetrical bell shape with a maximum centered at about two $\mathrm{eV} / 6 /$, and ii) the reemission angular distribution follows Lambert's law. Moreover, the re-emission coefficient $\delta$ is sensitive to the angle of incidence $\theta$ of the primaries and to the nature of the material considered. Consequently, topographic as well as material contrasts can be seen, and are superimposed on the possible voltage contrasts.

The backscattered emission coefficient $\eta$ is nearly constant in the energy domain considered, while the 
secondary emission $\delta(E p)$ takes on an asymmetrical bell shape 7,8 / versus the primary energy Ep. The maximum occurs in the keV range. Thus, when increasing Ep, the net charge deposited during SEM pictures achievement may start in the negative, becomes positive, and returns to the negative (fig.1). This is observed on insulators but it does not generally appear on metals $/ 3,4,9 /$. Besides, as we assume in the following, the re-emission coefficient $\delta$ is taken to be stable during the measurements, i.e. possible surface contamination effects are neglected.

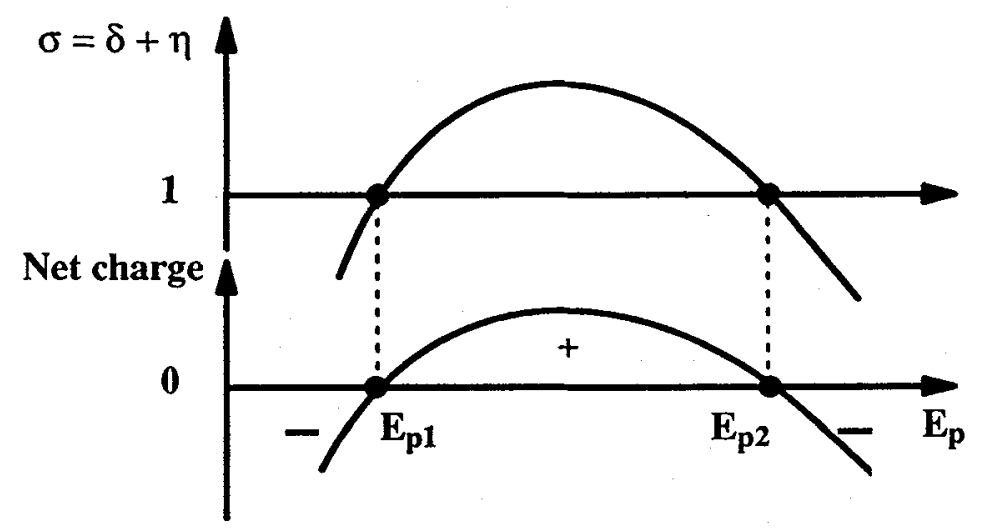

Fig. 1: Variation of: i) the total re-emission coefficient $\sigma=\delta+\eta$ and ii) the resulting net charge versus the primary energy Ep.

Due to the fact that microelectronic structures involve both surface and buried lines separated by insulators, it will be necessary to produce the SEM pictures when the insulator is under zero charging conditions /10/.

Thus the highest energy cross over Ep2 provides the most suitable conditions for good working conditions of electron optics and consequently for the production of clear pictures. Concerning the materials generally involved in microelectronics, Ep2 lies in the 0.5-2.5 keV range.

\section{III) Influence of local voltages on secondary electron signal.}

In order to clearly present the different physical phenomena underlying voltage contrast, we will split it into two cases where: i) static and, ii) dynamic or high frequency voltages are applied to the microelectronic structures.

\section{II-1 Static effect evaluations.}

On standard Scanning Electron Microscopes (SEMs) two electrostatic field configurations are found. Before reaching the detector the re-emitted electrons travel: i) underneath the objective lens, or ii) across the objective lens. So, above the sample, the distribution of electrostatic fields presents respectively: i) a plane, ii) an axis of symmetry. Thus, in both cases, a two dimensional space electrical simulation constitutes a suitable approximation.

The concept of the diagram of acceptance $D(e W, \theta)$ was developed some years ago/11/. Only given combina- 
tions of the initial energy (eW) and the angle of emission $(\theta)$ determine the acceptance area $A(e W, \theta)$. The re-emitted electrons belonging to it can reach the detector and consequently contribute to the secondary emission current. The acceptance area can be obtained through electrons trajectory simulations. In addition to the previous secondary electron characteristics, simulations must take into account the electrostatic field configuration above the sample. It depends on: i) the SEM chamber field distribution and ii) the voltage configuration of the test structure.

Typical voltage contrast test structures are made with three equidistant surface metal strips on insulator, they are parallel to the previously defined plane of symmetry. The applied voltages belong to the $\mathrm{O},+5 \mathrm{~V}$ range. The primary electrons impinge on the central conductor. The SE current is written:

$$
I_{s E}=\iint_{e W \theta} N(e W, \theta) \cdot \cos \theta \cdot d(e W) \cdot d(\theta)
$$

Let us consider a "below the lens" detector which corresponds also to low electrostatic fields inside the SEM chamber. Obviously, it gives a non symmetrical acceptance area, as sketched in fig. 2.

If a positive voltage $V s$ is applied to the central line, it gives rise to a potential barrier in the space above it. Low energy secondary electrons are re-attracted by this positive voltage line. Thus it is clear that the characteristic area is truncated between $\mathrm{eW}$ equals zero and approximately eVs (fig.2), especially for large angles of emission $\theta$. This results in the so called local field effect I (LFE I) $/ 11$ / which corresponds to the main voltage contrast effect observed in standard SEMs pictures.

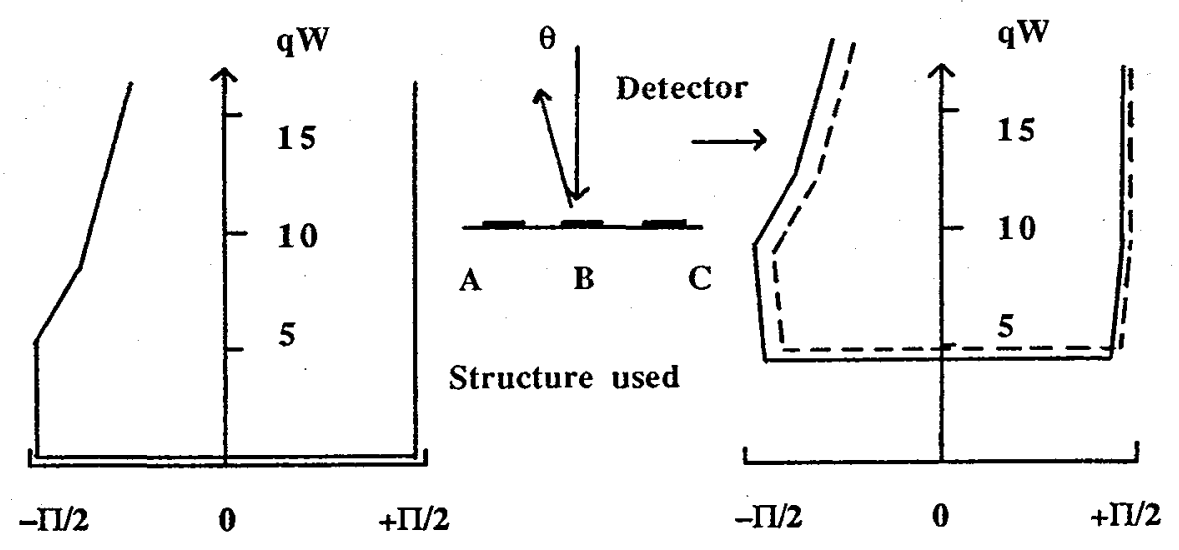

a)

b)

Fig. 2: Sketch of the acceptance diagrams in: a) absence, b) presence of the local field effects I (continuous line) and II (dotted line) for the three line structure voltages considered and a "below the lens" detector. In the case $b$ ), $a+5 \mathrm{~V}$ voltage is applied on the central line (B), while: $i$ ) in LFE I, other conductors

$(\mathrm{A}, \mathrm{C})$ are grounded and, ii) in LFE II, +5 and $0 \mathrm{~V}$ are applied to $\mathrm{A}$ and $\mathrm{C}$ respectively (after $/ 11 /)$.

If the adjacent lines bring different voltages, the electrons re-emitted near a positive voltage line are attracted, so the acceptance area is locally reduced and a dissymmetry is observed on it (fig.2). This point gives the local field effect II (LFE II) /11/ which may be responsible of cross talk effects in voltage measurements. 
Moreover, in the case of "above the lens" like secondary electron detection, a strong vertical extraction field (up to the $\mathrm{kV} / \mathrm{mm}$ ) may be available. Firstly, acceptance zones $\mathrm{A}(\mathrm{eW}, \theta)$ are symmetrical versus $\theta$. Secondly, the previous local barrier effects may be greatly reduced. So the acceptance diagram seen in the zero voltage case (fig. 2a) is not appreciably modified by local changes to a positive voltage. The result is a large reduction in both Local Field Effects (LFE) I and II.

In the ideal case, if the LFE are negligible, the ever present energy shifts eVs on the secondary electrons can be clearly detected. A high pass energy filtering allows the realization of the so called "S Curves" (fig.3). When no filtering occurs, all of the secondary electrons (SE) contribute to the current. In this case, it starts from a maximum and decreases to zero while increasing the threshold energy eVr of the filter. Filtering is generally carried out with a retarding field spectrometer $/ 12 \%$.

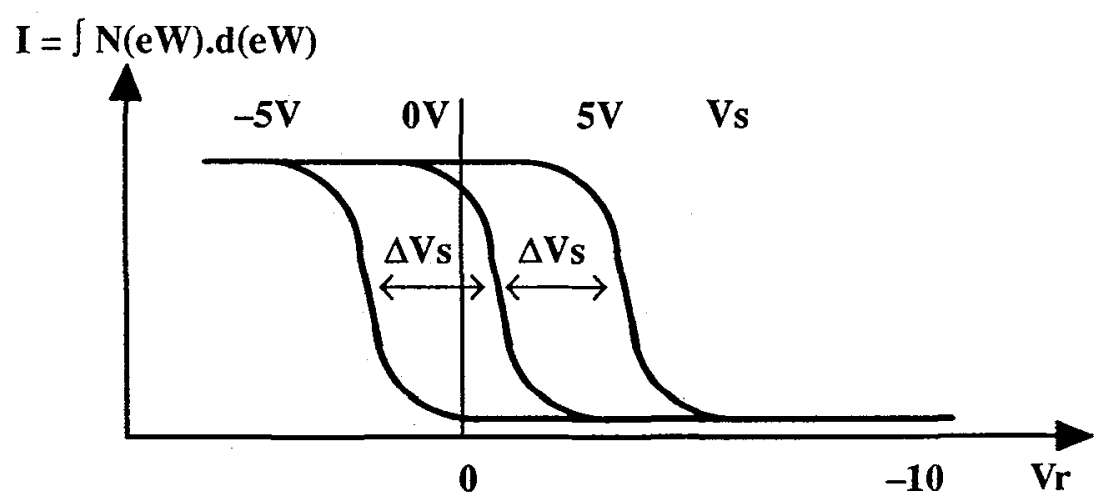

Fig. 3: Evolution of the detected SE currents ("S Curves") with target voltages Vs and retarding voltages Vr of a retarding field spectrometer.

Since Secondary Electrons (SE) can acquire an energy eVs, these "S Curves" are shifted by the same energy. If a shift $\Delta V r$ of the threshold voltage follows the corresponding target voltage shift $\Delta V s$, the $S E$ current is kept constant and $\Delta V_{I}=\Delta V_{T}$. This is the principle of electron beam voltage quantization. It is obtained by implementing a feedback loop in the retarding voltage circuit of the spectrometer $/ 12 /$. Of course, then the electron beam is no more scanned but focussed on the node of interest.

So, a minimum detectable voltage can be deduced from the signal over noise ratio $/ 13,14 /$, it becomes:

$$
V_{\min }=n \cdot C \sqrt{\frac{\Delta f}{I_{p e}}}
$$

It depends on: i) the admitted signal/noise ratio $\mathrm{n}$, ii) the sharpness of the energy cutting of the spectrometer which is included in $\mathrm{C}$, iii) the bandpass $\Delta f$ of the measurement apparatus, and iv) the primary beam current Ipe (in the $1 \mathrm{nA}$ range). Improvement about spectrometers /15,16/ have lead to the possibility of measuring differential voltages down to a few $\mathrm{mV}$.

Since: i) essentially low energy electrons are concerned by Local Field Effects (LFE), and ii) LFE are reduced in high extraction fields, the reduction of LFE can come close to the ideal case for surface lines widths down to 
one micrometer. Solutions include: i) eliminating very low energy SE, and ii) applying a strong extraction field (up to $2 \mathrm{kV} / \mathrm{mm}$ ) $/ 12 /$. However, as we shall see below, these conditions may not be generalized in all practical situations. But, using modern spectrometers, suitable accuracy results can be obtained.

\section{III-2 Dynamic voltage contrast.}

As strong need has been felt for the in situ observations of integrated circuits under normal working conditions, and, therefore, methods have been developed in order to meet this need. The principle one is based on the sampling of electrical signals. By this way, both voltage and temporal informations can be obtained. Since the physical events in SE reemission lie below the picosecond range/14/, the time accuracy mainly depends on: i) the width and jitter of the electron beam bursts impinging the examined structure, and, ii) the transit time effects (TTE). Of course, in any case the jitter must be much lower than the width of the sampling gate constituted by the burst of electrons. The consequences are observed on the voltage and time resolutions.

Many blanking techniques for the primary beam have been explored /18/. They allow us to overcome the bandpass of SE detectors which generally lie in the megahertz range. Of the equipments present on the market today, the most commonly available blankers are made up of two parallel plates deviating the primary beam inside or outside a diaphragm. However, due to the fact that the travelling electrons must present a maximum angle at the end of the blanker, frequency limitations do exist. The minimum width $T$ is of about $100 \mathrm{ps.}$ However it is sufficient for today's digital integrated circuits. The jitter must be consistent with the minimum beam pulse width, it is generally satisfied by modern electronics.

The transit time effect originates in the fact that, during the rise and decay parts of the voltages $V s(t)$ applied to the structure, the re-emitted $S E$ are subject to a local field changing with time $t$. This results in variations in the acceptance diagram $/ 19,20 \%$. In other words, the kinetic energy of the electrons arriving at the detector depends both on the $V s(t)$ at the time $t$ considered by the beam blanker, and also on the distribution of the local electrical fields $E(t)$ during the first part of their travel towards the detector $/ 21 /$. Thus a distortion of the "S Curves" occurs and a deformation of the voltages detected in transient regimes Vs(ton,toff) may be predicted $119,20,21,22,23 /$. But, as shown by electrical simulations, the spatial extent of the kinetic exchange zone around the re-emission point is about five times the tested line width. Thus it does not have a dramatic effect on very large scale integrated circuits results in which conductors lie down to the micrometer scale. But it must be kept in mind in the case of MMICs $/ 22 /$. Let us assume, for example, that we have a twenty ps ideal shape pulse and an extraction field of $2.5 \mathrm{kV} / \mathrm{mm}$. The corresponding pulse broadening or height reductions represent 10 per cent and less than 3 per cent for the extreme cases such as: i) a semi-infinite plane, or ii) a three line structure one micrometer wide $/ 23 /$.

The previous formulation of minimum detectable voltage is slightly modified /24/:

$$
V_{\min }-n \cdot C \cdot k \sqrt{\frac{1}{I_{p} \cdot T \cdot m}}
$$

and depends now on the beam pulse width $\mathrm{T}$ and the number $\mathrm{m}$ of burst averaging considered. Since, if we consider electron optics, the electron beam diameter increases with the beam current, a compromise must be found between the voltage, time and spatial resolutions required by the integrated circuit under examination. 
Since, in nowadays technologies, the number of conducting levels tends to increase and the ICs are covered with a passivation layer, it becomes of primary interest to obtain qualitative (logic levels) and quantitative electrical information about voltages carried out by conductors beneath insulators.

In the vertical device structures, the influence of a buried line voltage Vs extends up to the top insulator surface and the vacuum above. The proportion of the applied voltage Vs rising to this surface depends largely on the topographic structure under consideration. The previous three line configuration is then buried just below the surface. Then, locally induced surface voltages can easily be separated from signals induced by lateral lines, i.e. due to cross talk. Thus voltage quantization remains possible. In the case of lower buried levels the surface voltages are partially masked by upper conductors $/ 25 /$, so, essentially qualitative information can be obtained.

Under SEM electron beam irradiations, the top insulator can acquire either a negative or a positive voltage. The sign of the deposited charge depends on the material, the primary energy considered and the electrical field distribution in the SEM chamber. Since a negative charge deposition can induce a high surface charge $126 /$ damages to the IC may result as well as a deterioration in the clearness of the picture.

Conversely positive charging is a self limited process. Increasing the surface voltage charging causes more and more $S E$ to be reattracted to the surface. Thus a large decrease in secondary re-emission is observed, and the surface voltage quickly stabilizes to a few volts $/ 27,28,10 /$. This is a precondition for Capacitive Coupling Voltage Contrast (CCVC) observations.

Because an electrically exercised buried line induces a surface voltage change on a local level, a modulation of the Secondary Electron (SE) emission coefficient occurs on the top insulator. However, due to the continuous charge deposition during the achievement of the pictures, this local contrast is quickly cancelled. On the one hand, buried lines voltage contrast corresponds to "flashes" with similar grey level changes than those occurring in the case of surface conductors. On the other hand, the secondary electron energy shifts are driven by surface voltages. Thus, quantitative operation remains possible near the rise and decay edges of the electrical signals. Bearing in mind that the use of strong extraction fields -i.e. higher than $.1 \mathrm{kV} / \mathrm{mm}-$ appreciably changes the secondary emission coefficient $\delta / 28 /$, this solution should be avoided. Thus, specially designed electron optics and spectrometers, projecting a magnetic field onto the sample, improves the operation. So, surface line voltage contrast methods can generally be extended to buried lines $/ 29 /$.

\section{IV) Conditions for electron beam testing.}

The SEM parameters are: i) the primary beam energy $(.5-2.5 \mathrm{keV})$ and currents $(.1-10 \mathrm{nA})$, and ii) the extraction fields $(0-2 \mathrm{kV} / \mathrm{mm})$. They govern the spatial resolution and, in connection with the required time resolution in the blanked beam operation, the signal/noise ratio. However, by definition, electron beam testing must not be invasive for the integrated circuits. Moreover, if well controlled changes in the electrical characteristics of devices can be obtained, the use of the electron beam as an active probe can be anticipated.

Since MOS Integrated Circuits (ICs) are more sensitive to e-beam irradiations than bipolar circuits / $30 /$, we shall mainly discuss the case of MOS digital ICs.

The interaction of e-beam with semiconductors or insulators leads to the generation of electron hole pairs, 
plus the generation of characteristic $X$ Rays $/ 3,4 /$.

If a high energy beam (i.e. $10 \mathrm{keV}$ ) is used, then primary electrons can reach pn junctions, the result is an EBIC current. Therefore short circuiting of high logic levels is obtained $/ 31 /$ and might be used to activate logic levels inside ICs $/ 32 /$.

For moderate energies, $X$ Rays generated in the upper parts of a MOS structure can charge traps normally present inside the gate oxide of MOS transistors. The resultant threshold voltage shifts $/ 33,34 /$ depend on the couple primary energy-incident dose $/ 35 /$. This last is defined as the incident charge per unit of area falling on the circuit. But, since the sensitivity is largely dependent on the technology of ICs, to day this effect is not so dramatic in modern circuits (fig. 4).

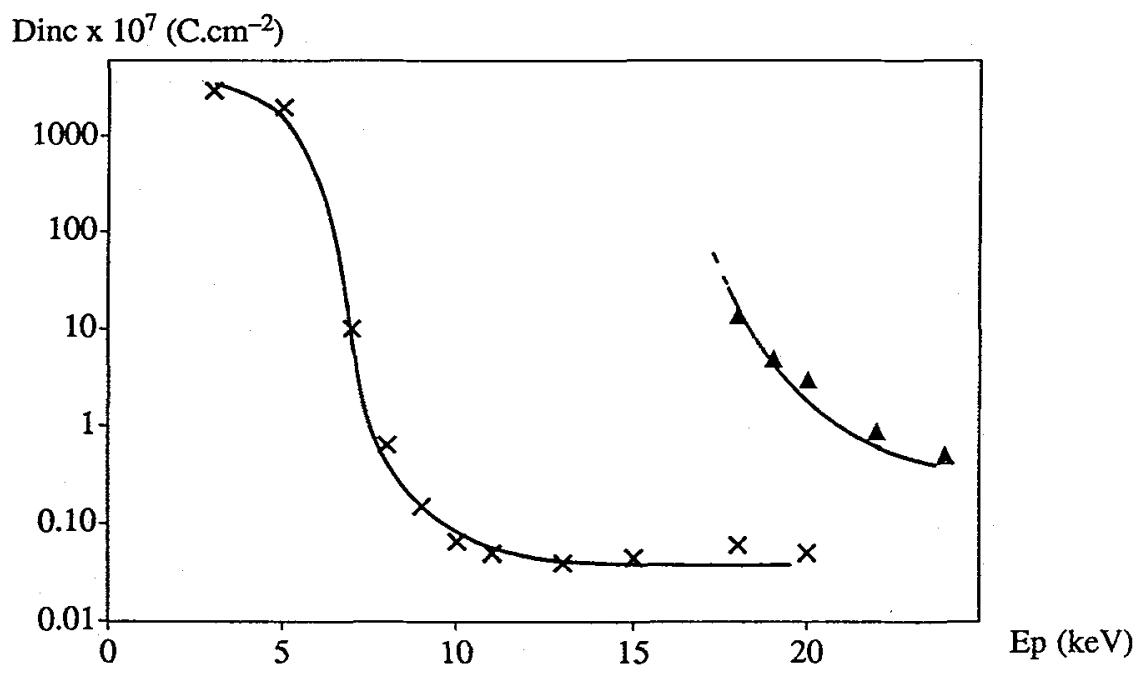

Fig. 4: Example of sensitivity to e-beam irradiations. The incident doses corresponding to a $.1 \mathrm{~V}$ threshold voltage shift are reported versus the primary energy Ep. The left and right hand side results correspond to different technologies i.e. with $600 \AA$ and $230 \AA$ gate oxide thicknesses respectively.

As previously reported $/ 36 /$, parasitic transistors can be generated by capacitive coupling with the charge deposited on the top insulator surface. Due to the pnpn structures often encountered in CMOS ICs, triggering of this thyristor like structure may be obtained during electron beam testing and, then, induce latch-up $/ 37 /$. If a standard MOS transistor is replaced by a floating gate device, the top charge directly influences the channel of the MOS transistor. This effect is at the origin of a method for charging yield measurements on insulators $/ 38 /$, and can be used as a switch structure $/ 39,40,41 /$ operating near or under the e-beam testing conditions. This is a way of achieving electron beam controllability of logic levels inside integrated circuits.

\section{V) E- beam testing techniques.}

The two main areas of voltage contrast applications concern the realization of pictures of a circuit's electrical state and the probe mode which allows the quantization of local voltage changes. 
This last point depends directly on the spectrometer, blanker and column characteristics. It is clear that vector injection the in input/output testing of ICs is faster than electron beam waveform measurements. Thus, local e-beam testing is generally introduced after an input/output defect has been found.

The various picture operating modes are related to the capabilities of the available equipment. Standard Scanning Electron Microscopes allow the examination of static or low frequency signals $/ 17,42 /$. The voltage resolution obtained is consistent with logic level operation. However, if a signal process including two parallel sample and hold chains $/ 43$ / is implemented on the standard signal chain, differential imaging becomes feasible. It allows: $i)$ the highlighting of the voltage contrast, ii) the obtention of a low cost, low speed blanking system.

The presence of a blanker allows us to overcome the standard SEM bandpass which is situated in the megahertz range. For example both stroboscopic and logic state imaging /17,44,45/ benefit from it.

Since, in failure analysis or in chip verification, the physical localization of a defect is not al ways easy, picture comparison techniques between "gold" and "failed" devices have been developed /46/. Moreover, a suitable selection of useful information such as frequency, phase or logical word related electrical signals considerably increases the speed of the operation $/ 47,48,49$. Automatic e-beam testing sequences combining picture and local voltage modes of operation have also been foreseen $/ 50 \%$

Today electron beam testers are linked to the integrated circuit database. So, chip layout and electrical simulations, which can be observed in connection with electron beam pictures, makes the integrated circuit designer's work easier. Moreover the automatic comparison between pictures and layout has been investigated $/ 51,52 /$. Modern integrated circuits include pre-designed test points devoted to electron beam testing operations /53/. Furthermore, ion milling /54/ and preferential electron beam etching /55/are likely to be of help in the creation of test points on buried lines which lie far beneath the surface.

\section{VI) The future of EB testing.}

The requirements of standard ICs are generally satisfied by the available dedicated e-beam testing equipment. But, due to the increasing operating speed of circuits a special demand has risen for CAD improvements and failure analysis. This domains concern: i) short time operations, i.e. the realization of extremely short sampling, and ii) automation in procedures.

We may notice that beam pulses of less than $10 \mathrm{ps}$ have been obtained with sophisticated blankers $/ 56,57 /$. Due to the fact that the direct current which is blanked lies in the nA range, the problem of the signal over noise ratio quickly becomes dramatic.

An alternative method consists in replacing of the standard thermoelectronic cathode by a photoemissive sheet. A high power, high energy $(\sim 5 \mathrm{eV})$ pulsed laser beam generates electron bursts which can extend down to $1.5 \mathrm{ps}$. The signal over noise ratio is then comparable with the performances of standard cathodes under $1 \mathrm{~ns}$ blanking /58/. Then the two voltage contrast modes of operation are retained.

Furthermore, direct probing using photoemission on the tested sample has been proposed and interesting results have been indicated $/ 59 \%$ 
Other local voltage or current probing methods have been evaluated. They use the fact that the presence of an electrical signal leads to changes in the refraction index inside $/ 60,61 /$ or in the vicinity of the $\mathrm{IC} / 62 /$. The result is a modulation in the intensity of a light with a lower than bandgap energy. This modulation is proportional to either the electrical field or the electrical current. Since modern lasers present pulse widths merging into the ps range, circuits such as MMICs are commonly probed. This method is feasible in devices implemented on either electro-optic or non electro-optic materials. Thus the way is open towards electrical measurements on circuits in the tens of Gigahertz range.

\section{VII) Conclusions.}

Since a lot of research has been carried out in recent years into voltage contrast, it is now becoming of a mature age. In this paper, we have examined the main physical aspects underlying voltage contrast. It allows both the realization of pictures of electrical states and local voltage quantizations on conductors which lie on the surface or buried under insulators. Various methods allow a suitable selection of characteristic electrical information. Thus, voltage contrast is now becoming a widely used tool in the design optimisation of innovative circuits and also in failure analysis groups.

If, to our knowledge, the performances obtained by commercial equipment are consistent with today's integrated circuits, the move towards future could be questioned. We have examined the possibilities and the limits of such a move. Furthermore, as we have illustrated, optical methods present a strong challenge to local electrical probing on Gigahertz range circuits.

\section{Acknowledgements.}

Our publications mentioned in this paper partly reflect the research fields which have been sponsored by CEC (ESPRIT Wafer Scale Integration no 824), GCIS (Groupement Circuit Intégrés Silicium) and CNET Centre Norbert Segard.

\section{References.}

/1/ G.S. Plows, W.C. Nixon, J. of Scientific Instrum. (J. of Phys. E), series 2, 1, 595, (1968).

/2/ C.W. Oatley, J. of Sc. Instr. ( J. of Phys. E), ser. 2, 2, 742, (1969).

13/ Microanalyse et microscopie electronique à balayage, Ecole d'été de St Martin d'Hères, Sept. 1978, Editions de Physique, Av. du Hoggar, 91944 Les Ullis cedex (France).

/4/ Scanning Electron Microscopy and X Rays Microanalysis, by J.I. Goldstein, D.E. Newbury, P. Echlin, D.C. Joy, C. Fiori, E. Lifshin, Plenum Press, 2nd ed., New York, (1984).

15/ F. Arnal, P. Verdier and P.D. Vincensini, C.R. Hebd. Acad. Sciences, 268, 1526, (1969).

/6/ R. Kollath, Handbuch der Physik, Springer Verlag, Germany, 21, 241, (1956).

7/ A.J. Dekker, Solid State Physics, 6, 251, F. Seitz, D. Turnbull eds, Academic Press, New York, (1958).

/8/H. Seiler, J. Appl. Phys 54(11), R1, (1983). 
19/ H.E. Bauer, H. Seiler, Scanning Electron Microscopy II, 1081, SEM Inc., Chicago (AMF O'Hare), USA, (1984).

/10/ P. Girard, P. Charpenel, P. Nouet, H. Martin, to be published in Proceedings of ESREF Conference, Bordeaux, Oct. 1991.

/11/ K. Nakamae, H. Fujioka, K. Ura, J. of Phys. D,: Appl. Phys., 14, 1939, (1981).

/12/ H.P. Feuerbaum, Scanning Electron Microscopy I, 285, SEM Inc., Om Johari ed., Chicago (AMF O'Hare), IL 60666, USA, (1979).

/13/ A. Gopinath, J. of Phys. E, E10, 911, (1977).

/14/ E. Menzel, E. Kubalek, Scanning 5, 103, (1983).

/15/ E. Menzel, R. Buchanan, Electronics Lett., 20, 10, 15th May 1984.

/16/ E. Plies, Microel. Eng., 12, 1-4, 189, (1990).

/17/ H.P. Feuerbaum, D. Kantz, E. Wolfgang, E. Kubalek, IEEE J. of Solid State Circuits, Vol. SC 13, 3, 319, June 1978.

/18/ E. Menzel, Scanning Electron Microscopy, I, 305, SEM Inc., Chicago (AMF O'Hare), IL 60666, USA, (1979).

/19/H. Fujioka, K. Nakamae, K. Ura, J. of Phys. D: Appl. Phys., 18, 1019, (1985).

/20/ K. Nakamae, H. Fujioka, K. Ura, Meas. Sci. Technol. 1, 894, (1990).

/21/R. Clauberg, J. of Appl. Physics 62(5), 1553, 1 Sept., 1987.

/22/ W. Mertin, K. D. Hermann, E. Kubalek, Microel. Eng. 12, 1, 287, (1990).

/23/ R. Clauberg, J. of Appl. Phys. 62(10), 4017, 15 Nov. 1987.

/24/H. Fujioka, K. Nakamae, K. Ura, J. Phys. E: Sci. Instrum., 18, 598, (1985).

125/ S. Goerlich, K.D. Herrmann, W. Reiners and E. Kubalek, Scanning Electron Microscopy II, 447, SEM Inc., Chicago (AMF O'Hare), IL 60666, USA, (1986).

/26/ D.M. Taylor, J. of Phys. E: Appl. Phys., 11, 2443, (1978).

127/ S. Goerlich, E. Kubalek, Scanning Electron Microscopy I, 81, SEM Inc., Chicago (AMF O'Hare), IL 60666, USA, (1985).

/28/ P. Girard, B. Pistoulet, M. Valenza, Journ. de Phys., Coll. C4, Sup. 9, T. 49, Sept. 1988.

129/ W. Reiners, Microelectronic Eng. 12, 325, (1990).

130/ K.A. Jenkins, IEEE Trans. on El. Devices, 36, 1722, (1989).

/31/ D.C. Shaver J. of Vac. Sc. and Techn. 19(4), 1010, (1981).

/32/ D. Micollet, B. Courtois, Microel. Eng., 7, 419, (1987).

/33/ K. Nakamae, H. Fujioka, K. Ura, J. Appl. Phys. 52(3), 1306, March 1981.

134/ S. Goerlich, E. Kubalek, Microel. Eng. 1, 93, (1983).

135/ P. Girard, Scanning Microscopy II, 1, 151, Scanning Microscopy International, Om Johari ed., Chicago (AMF O'Hare), IL 60666, USA, (1988).

136/ P. Girard, B. Pistoulet, P. Nouet, Ann. de Phys., Coll. 2, Sup. 6, Vol. 14, 253, Dec. 1989.

/37/ F.M. Roche, S.D. Bocus, P. Girard, ESSDERC Conference, Sept. 1991, Lausanne, to be published in the Proceedings. 
138/ P. Girard, Scanning Microscopy 2, 4, 1995, Scanning Microsc. Intern., Chicago (AMF O'Hare), II 60666, USA, (1988).

139/D.C. Shaver, J. Vac. Sci. Technol. B 1(4), 1084, Oct. Dec. 1984.

/40/ D.C. Shaver, IEEE Electr. Dev. Lett., EDL-4, 5, 153, May 1983.

141/ P. Girard, P. Nouet, B. Pistoulet, Microel. Eng. 12, 129 (1990).

142/ G.V. Lukianoff, T.R. Tow, Scanning Electron Micr. I, 465, (1975).

143/ J.P. Collin, T. Viacroze, Proc. of ISTFA, 89, Los Angeles 1985, ATFA ed., Torrance CAL 90510 , USA.

/44/ E. Wolfgang, R. Lindner, P. Fazekas, H.P. Feuerbaum, IEEE Trans. on El. Dev., ED 26, E, 549, (1979).

/45/ E. Menzel, E. Kubalek, Scanning Electron Microscopy I, 305, SEM Inc., Chicago (AMF O'Hare), IL 60666, USA, (1981).

146/ J.P. Collin, J.L. Pelissier, Microel. Eng., 7, 2-4, 385, (1987).

/47/ H.D. Brust, F. Fox, E. Wolfgang, Microelectronic Engineering 5, 411, (1985).

/48/ J.P. Collin, Revue de Phys. Appliquée,Coll. C6, Sup. no 6, T.24, C6-129, juin 1989.

/49/ H.D. Brust, F. Fox, Microel. Eng. 3, 191, (1985).

150/ M. Melgara, M. Battu, P. Garino, Microel. Eng. 12, 1-4, 269, (1990).

/51/ T. Tamama, N. Kuji, Design and Test of Computers,23, Aug. 1986.

152/ T.C. May, G.L. Scott, E.S. Meieran, P. Winer, V.S. Rao, Proceedings IEEE International Reliability Physics Symposium, 94, (1984).

153/ J. Kholzer, M. Killian, K. Althoff, F. Bonner, S. Goerlich, J. Otto, W. Argyro, F. Fox, H. Hemmert, D. Sommer, Microel. Eng., 12, 1-4, 27, (1990).

154/ D.C. Shaver, B.W. Ward, J. of VaC. Sc. and Techn. B, 4, 185, (1986).

155/ H. Fujioka, K. Nakamae, M. Hirota, K. Ura, N. Tamura, T. Takagi, J. of Phys. D: Appl. Phys., 23, 266, (1990).

156/ R. Schmitt, D. Winkler, M. Brunner, J.M. Dortu, Microelectr. Engineer. 12, 279, (1990).

157/ M. Brunner, R. Schmitt, D. Winkler, B. Lischke, Microel. Eng. 9, 405, (1989).

/58/ P. May, J.M. Halbout, C.T. Chuang, G.P. Li, IEEE Trans on EI. Dev., 35, 7, 1126, July 1988.

159/ A. Blacha, R. Clauberg,H.K. Seitz, J. Appl. Phys. 62(2), 713, 15 July 1987.

/60/ M.J.W. Rodwell, M. Riazat, K.J. Weingarten, B.A. Auld, D. Bloom IEEE Trans. on Micr. Theor. and Techn., MTT 34, 12, 1356, Dec. 1986.

/61/ B.R. Hemenway, H.K. Heinrich; J.H. Goll, D.M. Bloom, IEEE Electron Device Letters, EDL 8 , 8, 344, Aug. 1987.

/62/J.A. Valdmanis, G. Mourou, C.W. Gabel, Appl. Phys. Lett. 41(3), 211, 1st Aug. 1982. 